Algebraic $\& \mathcal{G}$ Geometric Topology

Volume 3 (2003) 187-205

Published: 22 February 2003

ATG

\title{
Realising formal groups
}

\author{
N.P. STRICKLAND
}

\begin{abstract}
We show that a large class of formal groups can be realised functorially by even periodic ring spectra. The main advance is in the construction of morphisms, not of objects.
\end{abstract}

AMS Classification $55 \mathrm{~N} 20 ; 55 \mathrm{~N} 22$

Keywords Generalized cohomology, formal group

\section{Introduction}

Let FG be the category of formal groups (of the sort usually considered in algebraic topology) over affine schemes. Thus, an object of FG consists of a pair $(G, S)$, where $S$ is an affine scheme, $G$ is a formal group scheme over $S$, and a coordinate $x$ can be chosen such that $\mathcal{O}_{G} \simeq \mathcal{O}_{S} \llbracket x \rrbracket$ as $\mathcal{O}_{S}$-algebras. A morphism from $\left(G_{0}, S_{0}\right)$ to $\left(G_{1}, S_{1}\right)$ is a commutative square

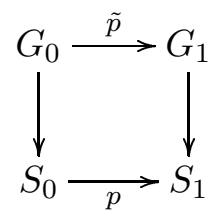

such that the induced map $G_{0} \rightarrow p^{*} G_{1}$ is an isomorphism of formal group schemes over $S_{0}$.

Next, recall that an even periodic ring spectrum is a commutative and associative ring spectrum $E$ such that $E^{1}=0$ and $E^{2}$ contains a unit (which implies that $E \simeq \Sigma^{2} E$ as spectra). Here we are using the usual notation $E^{k}=E^{k}$ (point) $=\pi_{-k} E$. We write EPR for the category of even periodic ring spectra. (Everything here is interpreted in Boardman's homotopy category of spectra; there are no $E_{\infty}$ or $A_{\infty}$ structures.)

Given an even periodic ring spectrum $E$, we can form the scheme $S_{E}:=$ $\operatorname{spec}\left(E^{0}\right)$ and the formal group scheme $G_{E}=\operatorname{spf}\left(E^{0} \mathbb{C} P^{\infty}\right)$ over $S_{E}$. This construction gives rise to a functor $\Gamma: \mathrm{EPR}^{\mathrm{op}} \rightarrow \mathrm{FG}$. 
It is a natural problem to try to define a realisation functor $R: \mathrm{FG} \rightarrow \mathrm{EPR}^{\mathrm{op}}$ with $\Gamma R(G, S) \simeq(G, S)$, or at least to do this for suitable subcategories of FG. For example, if we let LFG denote the category of Landweber exact formal groups, and put $\mathrm{LEPR}=\{E \in \mathrm{EPR} \mid \Gamma(E) \in \mathrm{LFG}\}$, one can show that the functor $\Gamma: \mathrm{LEPR}^{\mathrm{op}} \rightarrow \mathrm{LFG}$ is an equivalence; this is essentially due to Landweber, but details of this formulation are given in [5, Proposition 8.43]. Inverting this gives a realisation functor for LFG, and many well-known spectra are constructed using this. In particular, this gives various different versions of elliptic cohomology, based on various universal families of elliptic curves over rings such as $\mathbb{Z}\left[\frac{1}{6}, c_{4}, c_{6}\right]\left[\Delta^{-1}\right]$.

It is hard to say more than this unless we invert the prime 2 . We therefore make a blanket assumption:

Assumption 1.1 From now on, all rings are assumed to be $\mathbb{Z}\left[\frac{1}{2}\right]$-algebras. In particular, we only consider schemes $S$ for which 2 is invertible in $\mathcal{O}_{S}$. We use the symbol $M U$ for the spectrum that would normally be called $M U\left[\frac{1}{2}\right]$.

The other main technique for constructing realisations is the modernised version of Baas-Sullivan theory $[2,4]$. This starts with a strictly commutative ring spectrum $R$, and an algebra $A_{*}$ over $\pi_{*} R$, and it constructs a homotopically commutative $R$-algebra spectrum $A$ with $\pi_{*} A=A_{*}$, provided that $A_{*}$ has good structural properties. Firstly, we assume as always that 2 is invertible in $A_{*}$. Given this, the construction will work if $A_{*}$ is a localised regular quotient (LRQ) of $R_{*}$, in other words it has the form $A_{*}=\left(S^{-1} \pi_{*} R\right) / I$, where $S$ is a multiplicative set and $I$ is an ideal generated by a regular sequence. The construction can also be extended to cover the case where $A_{*}$ is a free module over an LRQ of $\pi_{*} R$.

We can apply this taking $R$ to be the periodic bordism spectrum

$$
M P=\bigvee_{n \in \mathbb{Z}} \Sigma^{2 n} M U\left[\frac{1}{2}\right]
$$

(we will verify in the appendix that this can be constructed as a strictly commutative ring). Given a formal group $(G, S)$ we can choose a coordinate $x$, which gives a formal group law $F$ defined over $\mathcal{O}_{S}$, and thus a ring map $\pi_{0} M P \rightarrow \mathcal{O}_{S}$, making $\mathcal{O}_{S}$ into a $\pi_{0} M P$-algebra. If this algebra has the right properties, then we can use the Baas-Sullivan approach to construct $E$ with $\Gamma(E) \simeq(G, S)$. It is convenient to make the following ad hoc definition:

Definition 1.2 A ring $R$ is standard if 2 is invertible in $R$ and $R$ is either a field or a ring of the form $T^{-1} \mathbb{Z}$ (for some set $T$ of primes). 
An easy argument given below shows that the above method can construct realizations for all formal groups over standard rings. Unfortunately, this construction is not obviously functorial: it depends on a choice of coordinate, and morphisms of formal groups do not generally preserve coordinates. The main result of this paper is to show that with suitable hypotheses we can nonetheless define a functor.

The basic point is to consider the situation where we have several different coordinates, say $x_{0}, \ldots, x_{r}$ on a fixed formal group $G$. In a well-known way, this makes $\mathcal{O}_{S}$ into an algebra over the ring $\pi_{0}\left(M P^{(r+1)}\right)$, and we can ask whether this can be realized topologically by an $M P^{(r+1)}$-algebra; the question will be made more precise in Section 3. We say that $G$ is very good if the question has an affirmative answer for all $r \geq 0$ and all $x_{0}, \ldots, x_{r}$.

Theorem 1.3 All formal groups over standard rings are very good.

This will be proved as Corollary 3.15.

For our sharpest results, we need a slightly more complicated notion. We say that a coordinate $x_{0}$ is multirealisable if for any list $x_{1}, \ldots, x_{r}$ of additional coordinates, the question mentioned above has an affirmative answer. We say that $G$ is good if it admits a multirealisable coordinate. Of course, $G$ is very good iff every coordinate is multirealisable. We write GFG for the category of good formal groups (considered as a full subcategory of FG). The details are given in Definition 3.12.

Theorem 1.4 Let $x$ be a coordinate on a formal group $(G, S)$, and suppose that the classifying map $\pi_{0} M P \rightarrow \mathcal{O}_{S}$ makes $\mathcal{O}_{S}$ into a localised regular quotient of $\pi_{0} M P$. Then $x$ is multirealisable, and so $G$ is good.

This will be proved as Proposition 3.14.

Corollary 1.5 At odd primes, the formal groups associated to 2-periodic versions of $B P, P(n), B(n), E(n), K(n), k(n)$ and so on are all good.

This shows that there is a considerable overlap with the Landweber exact case. However, there are many good formal groups that are not Landweber exact. Conversely, there is no reason to expect that Landweber exact formal groups will be good, although we have no counterexamples.

Our main result is as follows: 
Theorem 1.6 There is a realisation functor $R: \mathrm{GFG} \rightarrow \mathrm{EPR}$, with $\Gamma R \simeq$ 1: GFG $\rightarrow$ GFG.

Note that good formal groups are realisable by definition; the content of the theorem is that the realisation is well-defined and functorial.

We next explain the formal part of the construction; in Section 4 we will give additional details and prove that we have the required properties. The functor $R$ actually arises as $U V^{-1}$ for a pair of functors GFG $\stackrel{V}{\leftarrow} \stackrel{U}{\longrightarrow}$ EPR in which $V$ is an equivalence. To explain $\mathcal{E}$, recall that we have a topological category $\operatorname{Mod}_{0}$ of $M P$-modules. We write $\mathrm{DMod}_{0}$ for the derived category, and $\mathrm{EPA}_{0}$ for the category of even periodic commutative ring objects in $\operatorname{DMod}_{0}$. The unit map $\eta: S \rightarrow M P$ gives a functor $\eta^{*}: \mathrm{EPA}_{0} \rightarrow \mathrm{EPR}$, and the objects of the category $\mathcal{E}$ are the objects $E \in \mathrm{EPA}_{0}$ for which the associated coordinate on $\Gamma\left(\eta^{*} E\right)$ is multirealisable. The morphism set $\mathcal{E}\left(E_{0}, E_{1}\right)$ is a subset of $\operatorname{EPR}\left(\eta^{*} E_{0}, \eta^{*} E_{1}\right)$, the functor $V: \mathcal{E} \rightarrow$ GFG is given by $\Gamma$, and the functor $U: \mathcal{E} \rightarrow \mathrm{EPR}$ is given by $\eta^{*}$. We say that a map $f: \eta^{*} E_{0} \rightarrow \eta^{*} E_{1}$ in EPR is good if there is a commutative ring object $A$ in the derived category of $M P \wedge M P$-modules together with maps $f^{\prime}: E_{0} \rightarrow(1 \wedge \eta)^{*} A$ and $f^{\prime \prime}:(\eta \wedge 1)^{*} A \rightarrow E_{1}$ in $\mathrm{EPA}_{0}$ such that $f^{\prime \prime}$ is an equivalence and $f$ is equal to the composite

$$
\eta^{*} E_{0} \stackrel{\eta^{*} f^{\prime}}{\longrightarrow}(\eta \wedge \eta)^{*} A \stackrel{\eta^{*} f^{\prime \prime}}{\longrightarrow} \eta^{*} E_{1} .
$$

The morphisms in the category $\mathcal{E}$ are just the good maps. To prove Theorem 1.6, we need to show that

(3) The composite of two good maps is good, so $\mathcal{E}$ really is a category.

(2) For any map $\Gamma\left(\eta^{*} E_{0}\right) \rightarrow \Gamma\left(\eta^{*} E_{1}\right)$ of good formal groups, there is a unique good map $\eta^{*} E_{0} \rightarrow \eta^{*} E_{1}$ inducing it, so that $V$ is full and faithful.

(1) For any good formal group $(G, S)$ there is an object $E \in \mathrm{EPA}_{0}$ such that $\Gamma\left(\eta^{*} E\right) \simeq(G, S)$, so $V$ is essentially surjective.

To prove statement $(k)$, we need to construct modules over the $k$-fold smash power of $M P$. It will be most efficient to do this for all $k$ simultaneously.

\section{Preliminaries}

\subsection{Differential forms}

Let $(G, S)$ be a formal group, and let $I \leq \mathcal{O}_{G}$ be the augmentation ideal. Recall that the cotangent space of $G$ at zero is the module $\omega_{G}=I / I^{2}$. If $x$ 
is a coordinate on $G$ that vanishes at zero, then we write $d x$ for the image of $x$ in $I / I^{2}$, and note that $\omega_{G}$ is freely generated over $\mathcal{O}_{S}$ by $d x$. We define a graded ring $D(G, S)^{*}$ by

$$
D(G, S)^{k}= \begin{cases}0 & \text { if } k \text { is odd } \\ \omega_{G}^{\otimes(-k / 2)} & \text { if } k \text { is even. }\end{cases}
$$

Here the tensor products are taken over $\mathcal{O}_{S}$, and $\omega_{G}^{\otimes n}$ means the dual of $\omega_{G}^{\otimes|n|}$ when $n<0$. Where convenient, we will convert to homological gradings by the usual rule: $D(G, S)_{k}=D(G, S)^{-k}$.

Now let $E$ be an even periodic ring spectrum with $\Gamma(E)=(G, S)$. We then have $\mathcal{O}_{G}=E^{0} \mathbb{C} P^{\infty}$ and $I=\widetilde{E}^{0} \mathbb{C} P^{\infty}$ and one checks easily that the inclusion $S^{2}=\mathbb{C} P^{1} \rightarrow \mathbb{C} P^{\infty}$ gives an isomorphism $\omega_{G}=I / I^{2}=\widetilde{E}^{0} S^{2}=E^{-2}$. Using the periodicity of $E$, we see that this extends to a canonical isomorphism $D(\Gamma(E))^{*} \simeq E^{*}$.

It also follows from this analysis (or from more direct arguments) that a map $f: E_{0} \rightarrow E_{1}$ in EPR is a weak equivalence if and only if $\pi_{0} f$ is an isomorphism.

\subsection{Periodic bordism}

Consider the homology theory $M P_{*}(X)=M U_{*}(X) \otimes \mathbb{Z}\left[u, u^{-1}\right]$, where $u$ has homological degree 2 (and thus cohomological degree -2 ). This is represented by the spectrum $M P=\bigvee_{n \in \mathbb{Z}} \Sigma^{2 n} M U$, with an evident ring structure. It is well-known that $M U$ is an $E_{\infty}$ ring spectrum; see for example [3, Section IX]. It is also shown there that $M U$ is an $H_{\infty}^{2}$ ring spectrum, which means (as explained in [3, Remark VII.2.9]) that $M P$ is an $H_{\infty}$ ring spectrum; this is weaker than $E_{\infty}$ in theory, but usually equivalent in practise. As one would expect, $M P$ is actually an $E_{\infty}$ ring spectrum; a proof is given in the appendix. It follows from [2, Proposition II.4.3] that one can construct a model for $M P$ that is a strictly commutative ring spectrum (or "commutative $S$-algebra"). We may also assume that it is a cofibrant object in the category of all strictly commutative ring spectra.

For typographical convenience, we write $M P(r)$ for the $(r+1)$-fold smash power $M P \wedge \ldots \wedge M P$, which is again a strictly commutative ring. The spectra $M P(r)$ fit together into a cosimplicial object in the usual way; for example, we have three maps

$$
\eta \wedge 1 \wedge 1,1 \wedge \eta \wedge 1,1 \wedge 1 \wedge \eta: M P(0) \rightarrow M P(2) .
$$


In the category of strictly commutative ring spectra, the coproduct is the smash product. It follows formally that the smash product of cofibrant objects is cofibrant, so in particular the objects $M P(r)$ are all cofibrant.

For $r>0$, it is well-known that $\pi_{*} M U^{(r+1)}$ is a polynomial algebra over $\pi_{*} M U$ on countably many generators, and it follows that there is a noncanonical isomorphism

$$
\pi_{0} M P(r) \simeq \pi_{0} M P\left[x_{1}, x_{2}, \ldots\right]\left[x_{1}^{-1}, \ldots, x_{r}^{-1}\right] .
$$

There are $r+1$ obvious inclusions $M P \rightarrow M P(r)$. We can use these to push forward the standard generator of $M P^{0} \mathbb{C} P^{\infty}$, giving $r+1$ different coordinates on the formal group $\Gamma(M P(r))$. We denote these by $\widetilde{x}_{0}, \ldots, \widetilde{x}_{r}$.

\section{$2.3 \quad$ Groups and laws}

We now define a category $\mathrm{FG}_{r}$ as follows. The objects are systems

$$
\left(G, S, x_{0}, \ldots, x_{r}\right),
$$

where $(G, S)$ is a formal group and the $x_{i}$ are coordinates on $G$. The morphisms from $\left(G, S, x_{0}, \ldots, x_{r}\right)$ to $\left(H, T, y_{0}, \ldots, y_{r}\right)$ are the maps $(\tilde{p}, p):(G, S) \rightarrow(H, T)$ in FG for which $\tilde{p}^{*} y_{i}=x_{i}$ for all $i$. Note that given $p$, the map $\tilde{p}$ is determined by the fact that $\tilde{p}^{*} y_{0}=x_{0}$. Thus, the forgetful functor $\left(G, S, x_{0}, \ldots, x_{r}\right) \mapsto S$ (from $\mathrm{FG}_{r}$ to the category of affine schemes) is faithful.

We also write $\mathrm{Alg}_{r}$ for the category of commutative algebras over the ring $\pi_{0} M P(r)$.

Proposition 2.1 There is an equivalence $\mathrm{FG}_{r} \simeq \mathrm{Alg}_{r}^{\mathrm{op}}$.

Proof Recall that we have coordinates $\widetilde{x}_{0}, \ldots, \widetilde{x}_{r}$ on $\Gamma(M P(r))$. Given an object $A \in \operatorname{Alg}_{r}$ we have a structure map $\operatorname{spec}(A) \rightarrow \operatorname{spec}\left(\pi_{0} M P(r)\right)$, and we can pull back $\Gamma(M P(r))$ to get a formal group $G_{A}$ over $\operatorname{spec}(A)$. We can also pull back the coordinates $\widetilde{x}_{i}$ to make $G_{A}$ an object of $\mathrm{FG}_{r}$. It is easy to see that this construction defines a functor $U: \operatorname{Alg}_{r}^{\text {op }} \rightarrow \mathrm{FG}_{r}$. By forgetting down to the category of affine schemes, we see that $U$ is faithful.

We now claim that $U$ is an equivalence. We will deduce this from a well-known result of Quillen by a sequence of translations. First, Quillen tells us that maps $\pi_{*} M U^{(r+1)} \rightarrow B_{*}$ of graded rings biject naturally with systems

$$
F_{0} \stackrel{f_{0}}{\longleftarrow} F_{1} \stackrel{f_{1}}{\longleftarrow} \cdots \stackrel{f_{r-1}}{\longleftarrow} F_{r},
$$


where each $F_{i}$ is a homogeneous formal group law over $B_{*}$ and each $f_{i}$ is a strict isomorphism. By a standard translation to the even periodic case, we see that maps $\pi_{0} M P(r) \rightarrow A$ of ungraded rings biject naturally with systems

$$
F_{0} \stackrel{f_{0}}{\longleftarrow} F_{1} \stackrel{f_{1}}{\longleftarrow} \cdots \stackrel{f_{r-1}}{\longleftarrow} F_{r},
$$

where each $F_{i}$ is a formal group law over $A$ and each $f_{i}$ is a (not necessarily strict) isomorphism.

Now suppose we have an object $\left(G, S, x_{0}, \ldots, x_{r}\right)$ in $\mathrm{FG}_{r}$. For each $i$ there is a unique formal group law $F_{i}$ over $\mathcal{O}_{S}$ such that $x_{i}(a+b)=F_{i}\left(x_{i}(a), x_{i}(b)\right)$ for sections $a, b$ of $G$. Moreover, as $x_{i+1}$ is another coordinate, we can write $x_{i}=f_{i}\left(x_{i+1}\right)$ for a unique power series $f_{i} \in \mathcal{O}_{S} \llbracket t \rrbracket$. It is easy to check that $f_{i}$ is an isomorphism from $F_{i+1}$ to $F_{i}$, so Quillen's theorem gives us a map $\pi_{0} M P(r) \rightarrow \mathcal{O}_{S}$, allowing us to regard $\mathcal{O}_{S}$ as an object of $\mathrm{Alg}_{r}$. It is easy to see that this construction gives a functor $\mathrm{FG}_{r} \rightarrow \mathrm{Alg}_{r}^{\mathrm{op}}$. We leave it to the reader to check that this is inverse to $U$.

\subsection{Module categories}

We write $\operatorname{Mod}_{r}$ for the category of $M P(r)$-modules (in the strict sense, not the homotopical one). Note that a map $f: A_{0} \rightarrow A_{1}$ of strictly commutative ring spectra gives a functor $f^{*}: \operatorname{Mod}_{A_{1}} \rightarrow \operatorname{Mod}_{A_{0}}$, which is just the identity on the underlying spectra (and thus preserves weak equivalences). It follows easily that for any two maps $A_{0} \stackrel{f}{\rightarrow} A_{1} \stackrel{g}{\rightarrow} A_{2}$, the functor $f^{*} g^{*}$ is actually equal (not just naturally isomorphic or naturally homotopy equivalent) to $(g f)^{*}$. Thus, the categories $\operatorname{Mod}_{r}$ fit together to give a simplicial category $\operatorname{Mod}_{*}$.

Remark 2.2 For us, a simplicial category means a simplicial object in the category of categories. Elsewhere in the literature, the same phrase is sometimes used to refer to categories enriched over the category of simplicial sets, which is a rather different notion.

Next, we write $\operatorname{DMod}_{r}$ the derived category of $\operatorname{Mod}_{r}$, as in [2, Chapter III]. As usual, there are two different models for a category such as $\operatorname{DMod}_{r}$ :

(a) One can take the objects to be the cofibrant objects in $\operatorname{Mod}_{r}$, and morphisms to be homotopy classes of maps; or

(b) One can use all objects in $\operatorname{Mod}_{r}$ and take morphisms to be equivalence classes of "formal fractions", in which one is allowed to invert weak equivalences. 
We will use model (b). This preserves the strong functorality mentioned previously, and ensures that $\operatorname{DMod}_{*}$ is again a simplicial category.

We also write $\mathrm{EPA}_{r}$ for the category of even periodic commutative ring objects in $\operatorname{DMod}_{r}$, giving another simplicial category. (Note that periodicity is actually automatic, because $M P(r)$ is itself periodic.) Various fragments of the simplicial structure will be used in Section 4.

\section{Basic realisation results}

Let $R$ be a strictly commutative ring spectrum that is even and periodic, such that $R_{0}$ is an integral domain (and as always, 2 is invertible). The main examples will be $R=M P(r)$ for $r \geq 0$. Let $\mathcal{D}$ be the derived category of $R$-modules, and let $\mathcal{R}$ be the category of commutative ring objects $A \in \mathcal{D}$ such that $\pi_{1} A=0$. Recall that if $f$ is a morphism in $\mathcal{R}$ such that $\pi_{0} f$ is an isomorphism, then $\pi_{*} f$ is also an isomorphism and so $f$ is an equivalence.

We also write $\mathcal{R}_{0}$ for the category of commutative algebras over $\pi_{0} R$. We say that an object $A \in \mathcal{R}$ is strong if for all $B \in \mathcal{R}$, the map

$$
\pi_{0}: \mathcal{R}(A, B) \rightarrow \mathcal{R}_{0}\left(\pi_{0} A, \pi_{0} B\right)
$$

is a bijection. A realisation of an object $A_{0} \in \mathcal{R}_{0}$ is a pair $(A, u)$, where $A \in \mathcal{R}$ and $u: \pi_{0} A \rightarrow A_{0}$ is an isomorphism. We say that $(A, u)$ is a strong realisation iff the object $A$ is strong; if so, we have a natural isomorphism $\mathcal{R}(A, B) \simeq \mathcal{R}_{0}\left(A_{0}, \pi_{0} B\right)$. We say that $A_{0}$ is strongly realisable if it admits a strong realisation. If so, it is easy to check that all realisations are strong, and any two realisations are linked by a unique isomorphism.

The results of [4] provide a good supply of strongly realisable algebras, except that we need a little translation between the even periodic framework and the usual graded framework. Suppose that $A_{0} \in \mathcal{R}_{0}$, and put $T=\operatorname{spec}\left(A_{0}\right)$. We have a unit map $\eta: \pi_{0} R \rightarrow A_{0}$ and thus a map $\operatorname{spec}(\eta): T \rightarrow S_{R}$; we can pull back the formal group $G_{R}$ along this to get a formal group $H:=\operatorname{spec}(\eta)^{*} G_{R}$ over $T$. From this we get a map $\eta_{*}: R_{*}=D\left(G_{R}, S_{R}\right)_{*} \rightarrow D(H, T)_{*}$, which agrees with $\eta$ in degree zero. Indeed, if we choose a generator $u$ of $R_{2}$ over $R_{0}$, then $\eta_{*}$ is just the map $R_{0}\left[u, u^{-1}\right] \rightarrow A_{0}\left[u, u^{-1}\right]$ obtained in the obvious way from $\eta$. It is easy to check that $A_{0}$ is strongly realisable (as defined in the previous paragraph) iff $D(H, T)_{*}$ is strongly realisable over $R_{*}$ (as defined in [4]). 
Definition 3.1 A short ordinal is an ordinal $\lambda$ of the form $n . \omega+m$ for some $n, m \in \mathbb{N}$. A regular sequence in a ring $R_{0}$ is a system of elements $\left(x_{\alpha}\right)_{\alpha<\lambda}$ for some short ordinal $\lambda$ such that $x_{\alpha}$ is not a zero-divisor in the ring $\left(S^{-1} R_{0}\right) /\left(x_{\beta} \mid \beta<\alpha\right)$. An object $A_{0} \in \mathcal{R}_{0}$ is a localised regular quotient (or LRQ) of $R_{0}$ if $A_{0}=\left(S^{-1} R_{0}\right) / I$ for some subset $S \subset R_{0}$ and some ideal $I \leq S^{-1} R_{0}$ that can be generated by a regular sequence.

Remark 3.2 We have made a small extension of the usual notion of a regular sequence, to ensure that any LRQ of an LRQ of $R_{0}$ is itself an LRQ of $R_{0}$; see Lemma 3.8 .

Proposition 3.3 If $A_{0}$ is an $L R Q$ of $R_{0}$, then it is strongly realisable.

Proof This is essentially [4, Theorem 2.6], translated into a periodic setting as explained above. Here we are using a slightly more general notion of a regular sequence, but all the arguments can be adapted in a straightforward way. The main point is that any countable limit ordinal has a cofinal sequence, so homotopy colimits can be constructed using telescopes in the usual way. Andrey Lazarev has pointed out a lacuna in [4]: it is necessary to assume that the elements $x_{\alpha}$ are all regular in $S^{-1} R_{0}$ itself, which is not generally automatic. However, we are assuming that $R_{0}$ is an integral domain so this issue does not arise.

Proposition 3.4 Suppose that

- $A$ and $B$ are strong realisations of $A_{0}$ and $B_{0}$

- The natural map $A_{0} \otimes_{R_{0}} B_{0} \rightarrow\left(A \wedge_{R} B\right)_{0}$ is an isomorphism.

Then $A \wedge_{R} B$ is a strong realisation of $A_{0} \otimes_{R_{0}} B_{0}$.

Proof This follows from [4, Corollary 4.5].

Proposition 3.5 If $A_{0} \in \mathcal{R}_{0}$ is strongly realisable, and $B_{0}$ is an algebra over $A_{0}$ that is free as a module over $A_{0}$, then $B_{0}$ is also strongly realisable.

Proof This follows from [4, Proposition 4.13].

Proposition 3.6 Suppose that $R_{0}$ is a polynomial ring in countably many variables over $\mathbb{Z}\left[\frac{1}{2}\right]$, that $A_{0} \in \mathcal{R}_{0}$, and that $A_{0}=\mathbb{Z}[1 / 2 n]$ as a ring (for some $n)$. Then $A_{0}$ is an $L R Q$ of $R_{0}$, and thus is strongly realisable. 
Proof Choose a system of polynomial generators $\left\{x_{k} \mid k \geq 0\right\}$ for $R_{0}$ over $\mathbb{Z}\left[\frac{1}{2}\right]$. Put $a_{k}=\eta\left(x_{k}\right) \in A_{0}=\mathbb{Z}[1 / n]$ and $y_{k}=x_{k}-a_{k} \in R_{0}[1 / n]$. It is clear that $R_{0}[1 / 2 n]=\mathbb{Z}[1 / 2 n]\left[y_{k} \mid k \geq 0\right]$, that the elements $y_{k}$ form a regular sequence generating an ideal $I$ say, and that $A_{0}=R_{0}[1 / 2 n] / I$.

Proposition 3.7 Suppose that $R_{0}$ is a polynomial ring in countably many variables over $\mathbb{Z}\left[\frac{1}{2}\right]$, that $A_{0} \in \mathcal{R}_{0}$, and that $A_{0}$ is a field (necessarily of characteristic different from 2). Then $A_{0}$ is a free module over an $L R Q$ of $R_{0}$, and thus is strongly realisable.

Proof For notational simplicity, we assume that $A_{0}$ has characteristic $p>2$; the case of characteristic 0 is essentially the same.

Choose a set $X$ of polynomial generators for $R_{0}$ over $\mathbb{Z}\left[\frac{1}{2}\right]$. Let $K$ be the subfield of $A_{0}$ generated by the image of $\eta$, or equivalently by $\eta(X)$. We can choose a subset $Y \subseteq X$ such that $\eta(Y)$ is a transcendence basis for $K$ over $\mathbb{F}_{p}$. This means that the subfield $L_{0}$ of $K$ generated by $\eta(Y)$ is isomorphic to the rational function field $\mathbb{F}_{p}(Y)$, and that $K$ is algebraic over $L_{0}$. Put $S=\mathbb{Z}\left[\frac{1}{2}, Y\right] \backslash\left(p \mathbb{Z}\left[\frac{1}{2}, Y\right]\right)$, so $L_{0}=\left(S^{-1} \mathbb{Z}\left[\frac{1}{2}, Y\right]\right) / p$. Next, list the elements of $X \backslash Y$ as $\left\{x_{1}, x_{2}, \ldots\right\}$, and let $L_{k}$ be the subfield of $K$ generated by $\left\{x_{i} \mid i \leq k\right\}$. (We will assume that $X \backslash Y$ is infinite; if not, the notation changes slightly.) As $x_{k}$ is algebraic over $L_{k-1}$, there is a monic polynomial $f_{k}(t) \in L_{k-1}[t]$ with $L_{k}=L_{k-1}\left[x_{k}\right] / f_{k}\left(x_{k}\right)$. As $L_{k-1}$ is a quotient of the ring $P_{k-1}:=S^{-1} \mathbb{Z}\left[Y, x_{1}, \ldots, x_{k-1}\right]$, we can choose a monic polynomial $g_{k}(t) \in P_{k-1}[t]$ lifting $f_{k}$, and put $z_{k}:=g_{k}\left(x_{k}\right) \in P_{k} \subseteq S^{-1} R_{0}$. It is not hard to check that the sequence $\left(p, z_{1}, z_{2}, \ldots\right)$ is regular in $S^{-1} R_{0}$, and that $\left(S^{-1} R_{0}\right) /\left(z_{i} \mid i>0\right)=K$, so $K$ is an LRQ of $R_{0}$. It is clear that $A_{0}$ is free over the subfield $K$.

Lemma 3.8 An $L R Q$ of an $L R Q$ is an $L R Q$.

Proof Suppose that $B=\left(S^{-1} A\right) /\left(x_{\alpha} \mid \alpha<\lambda\right)$ and $C=\left(T^{-1} B\right) /\left(y_{\beta} \mid \beta<\right.$ $\mu$ ), where $\lambda$ and $\mu$ are short ordinals, and the $x$ and $y$ sequences are regular in $S^{-1} A$ and $T^{-1} B$ respectively. Let $T^{\prime}$ be the set of elements of $A$ that become invertible in $T^{-1} B$; clearly $S \subseteq T^{\prime}$ and $T^{-1} B=\left(\left(T^{\prime}\right)^{-1} A\right) /\left(x_{\alpha} \mid \alpha<\lambda\right)$. As $\left(T^{\prime}\right)^{-1} A$ is a localisation of $S^{-1} A$ and localisation is exact, we see that $x$ is a regular sequence in $\left(T^{\prime}\right)^{-1} A$ as well. After multiplying by suitable elements of $T^{\prime}$ if necessary, we may assume that $y_{\beta}$ lies in the image of $A$ (this does not affect regularity, as the elements of $T^{\prime}$ are invertible). We then put $z_{\alpha}=$ $x_{\alpha}$ for $\alpha<\lambda$, and let $z_{\lambda+\beta}$ be any preimage of $y_{\beta}$ in $A$ for $0 \leq \beta<\mu$. 
This gives a regular sequence in $\left(T^{\prime}\right)^{-1} A$ indexed by $\lambda+\mu$, such that $C=$ $\left(\left(T^{\prime}\right)^{-1} A\right) /\left(z_{\gamma} \mid \gamma<\lambda+\mu\right)$ as required.

We now specialize to the case $R=M P(r)$, so $\mathcal{R}_{0}=\mathrm{EPA}_{r}$. We write $\Gamma_{r}$ for the evident composite functor

$$
\mathrm{EPA}_{r}^{\mathrm{op}} \stackrel{\pi_{0}}{\longrightarrow} \mathrm{Alg}_{r}^{\mathrm{op}} \simeq \mathrm{FG}_{r} .
$$

Translating our previous definitions via the equivalence $\mathrm{Alg}_{r}^{\mathrm{op}} \simeq \mathrm{FG}_{r}$, we obtain the following.

Definition 3.9 An object $A \in \mathrm{EPA}_{r}$ is strong if for all $B \in \mathrm{EPA}_{r}$, the map

$$
\Gamma_{r}: \operatorname{EPA}_{r}(A, B) \rightarrow \mathrm{FG}_{r}\left(\Gamma_{r}(B), \Gamma_{r}(A)\right)
$$

is a bijection.

Definition 3.10 A realisation of an object $(G, S, \underline{x}) \in \mathrm{FG}_{r}$ is a triple $(A, \tilde{p}, p)$, where $A \in \mathrm{EPA}_{r}$ and $(\tilde{p}, p): \Gamma_{r} A \rightarrow(G, S, \underline{x})$ is an isomorphism. This is a strong realisation if the object $A$ is strong.

We now give more precise versions of the definitions in the introduction.

Definition 3.11 A formal group $(G, S)$ is very good if for every nonempty list $\underline{x}$ of coordinates, the object $(G, S, \underline{x}) \in \mathrm{FG}_{r}$ is strongly realisable.

Definition 3.12 A coordinate $x_{0}$ on $G$ is multirealisable if for every list $x_{1}, \ldots, x_{r}$ of coordinates, the object $\left(G, S, x_{0}, \ldots, x_{r}\right) \in \mathrm{FG}_{r}$ is strongly realisable. A formal group $(G, S)$ is good if it admits a multirealisable coordinate. We write GFG for the category of good formal groups.

Remark 3.13 Let $x_{0}, \ldots, x_{r}$ be coordinates, and suppose that $x_{0}$ is multirealisable. Let $\sigma$ be a permutation of $\{0, \ldots, r\}$. Using the evident action of permutations on $M P(r)$, we see that the object $\left(G, S, x_{\sigma(0)}, \ldots, x_{\sigma(r)}\right)$ is strongly realisable.

Proposition 3.14 Suppose that $x_{0}$ is such that the classifying map $\pi_{0} M P \rightarrow$ $\mathcal{O}_{S}$ makes $\mathcal{O}_{S}$ an LRQ of $\pi_{0} M P$. Then $x_{0}$ is multirealisable, so $(G, S)$ is good. 
Proof The coordinate $x_{0}$ gives a map $f_{0}: \pi_{0} M P \rightarrow \mathcal{O}_{S}$. By assumption, there is a multiplicative set $T \subseteq \pi_{0} M P$ and a regular ideal $I$ such that $f_{0}$ induces an isomorphism $\left(T^{-1} \pi_{0} M P\right) / I \rightarrow \mathcal{O}_{S}$.

Now consider a list of additional coordinates $x_{1}, \ldots, x_{r}$ say. These give a map $f: \pi_{0} M P(r) \rightarrow \mathcal{O}_{S}$ extending $f_{0}$. We know from Section 2.2 that $\pi_{0} M P(r)$ is a polynomial ring in countably many variables over $\pi_{0} M P$, in which $r$ of the variables have been inverted, so we can write

$$
\pi_{0} M P(r)=\pi_{0} M P\left[u_{1}, u_{2}, \ldots\right]\left[u_{1}^{-1}, \ldots, u_{r}^{-1}\right] .
$$

Put

$$
A_{0}=\mathcal{O}_{S}\left[u_{1}, u_{2}, \ldots\right]\left[u_{1}^{-1}, \ldots, u_{r}^{-1}\right],
$$

which is evidently an LRQ of $\pi_{0} M P(r)$. It is easy to see that $f$ induces a map $f^{\prime}: A_{0} \rightarrow \mathcal{O}_{S}$ of $\mathcal{O}_{S}$-algebras. Put $a_{k}=f^{\prime}\left(u_{k}\right) \in \mathcal{O}_{S}$, and $v_{k}=u_{k}-a_{k} \in A_{0}$. Clearly $A_{0}$ is a localisation of $\mathcal{O}_{S}\left[v_{k} \mid k>0\right]$, the sequence of $v$ 's is regular in $A_{0}$, and $A_{0} /\left(v_{k} \mid k>0\right)=\mathcal{O}_{S}$ as $\pi_{0} M P(r)$-algebras. It follows that $\mathcal{O}_{S}$ is an LRQ of an LRQ, and thus an LRQ, over $\pi_{0} M P(r)$. It is thus strongly realisable as required.

Corollary 3.15 If $\mathcal{O}_{S}$ is a standard ring, then every coordinate is multirealisable, and so $(G, S)$ is very good.

Proof This now follows from Propositions 3.6 and 3.7.

\section{Proof of the main theorem}

Let $\mathcal{E}$ denote the class of objects $E \in \mathrm{EPA}_{0}$ for which the resulting coordinate on $\Gamma\left(\eta^{*} E\right)$ is multirealisable. Note that this means that $\Gamma_{1} E$ is strongly realisable, so every realisation is strong, so in particular $E$ is a strong object.

Proposition 4.1 For any good formal group $(G, S)$, there exists $E \in \mathcal{E}$ with $\Gamma\left(\eta^{*} E\right) \simeq(G, S)$.

Proof By the definition of goodness we can choose a multirealisable coordinate $x_{0}$ on $G$. This means in particular that the object $\left(G, S, x_{0}\right) \in \mathrm{FG}_{0}$ is isomorphic to $\Gamma_{0}(E)$ for some $E \in \mathrm{EPA}_{0}$. It follows that $(G, S) \simeq \Gamma\left(\eta^{*} E\right)$, as required. 
Proposition 4.2 Suppose we have objects $E_{0}, E_{1} \in \mathcal{E}$, together with a map

$$
(\tilde{p}, p): \Gamma\left(\eta^{*} E_{1}\right) \rightarrow \Gamma\left(\eta^{*} E_{0}\right)
$$

in GFG. Then there is a unique good map $f: \eta^{*} E_{0} \rightarrow \eta^{*} E_{1}$ such that $\Gamma(f)=$ $(\tilde{p}, p)$.

Proof We first put $\left(G_{i}, S_{i}, x_{i}\right)=\Gamma_{0} E_{i}$ for $i=0,1$.

We introduce a category $\mathcal{B}=\mathcal{B}\left(E_{0}, E_{1}, \tilde{p}, p\right)$ as follows. The objects are triples $\left(A, f^{\prime}, f^{\prime \prime}\right)$ where

(a) $A$ is an object of $\mathrm{EPA}_{1}$.

(b) $f^{\prime}$ is a morphism $E_{0} \rightarrow(1 \wedge \eta)^{*} A$ in $\mathrm{EPA}_{0}$.

(c) $f^{\prime \prime}$ is an isomorphism $(\eta \wedge 1)^{*} A \rightarrow E_{1}$ in $\mathrm{EPA}_{0}$.

(d) The composite

$$
f=\theta\left(A, f^{\prime}, f^{\prime \prime}\right):=\left(\eta^{*} E_{0} \stackrel{\eta^{*} f^{\prime}}{\longrightarrow}(\eta \wedge \eta)^{*} A \stackrel{\eta^{*} f^{\prime \prime}}{\longrightarrow} \eta^{*} E_{1}\right)
$$

satisfies $\Gamma(f)=(\tilde{p}, p)$.

The morphisms from $\left(A, f^{\prime}, f^{\prime \prime}\right)$ to $\left(B, g^{\prime}, g^{\prime \prime}\right)$ in $\mathcal{B}$ are the isomorphisms $u: A \rightarrow$ $B$ in $\mathrm{EPA}_{1}$ for which $\left((1 \wedge \eta)^{*} u\right) f^{\prime}=g^{\prime}$ and $g^{\prime \prime}\left((\eta \wedge 1)^{*} u\right)=f^{\prime \prime}$.

The maps of the form $\theta\left(A, f^{\prime}, f^{\prime \prime}\right)$ are precisely the good maps that induce $(\tilde{p}, p)$, and isomorphic objects of $\mathcal{B}$ have the same image under $\theta$. It will thus suffice to show that $\mathcal{B} \neq \emptyset$ and all objects of $\mathcal{B}$ are isomorphic.

First, as $x_{1}$ is multirealisable, we can find an object $A \in \mathrm{EPA}_{1}$ and an isomorphism $(\tilde{q}, q): \Gamma_{1} A \rightarrow\left(G_{1}, S_{1}, \tilde{p}^{*} x_{0}, x_{1}\right)$ displaying $A$ as a strong realisation of $\left(G_{1}, S_{1}, \tilde{p}^{*} x_{0}, x_{1}\right)$. We write $\left(H, T, y_{0}, y_{1}\right)=\Gamma_{1} A$, so $(\tilde{q}, q):(H, T) \stackrel{\simeq}{\longrightarrow}\left(G_{1}, S_{1}\right)$ and $(\tilde{p} \tilde{q})^{*} x_{0}=y_{0}$ and $\tilde{q}^{*} x_{1}=y_{1}$. We can thus regard $(\tilde{p} \tilde{q}, p q)$ as a morphism

$$
\Gamma_{0}\left((1 \wedge \eta)^{*} A\right)=\left(H, T, y_{0}\right) \rightarrow\left(G_{0}, S_{0}, x_{0}\right)=\Gamma_{0} E_{0},
$$

and $E_{0}$ is a strong realisation of $\left(G_{0}, S_{0}, x_{0}\right)$, so this must come from a map $f^{\prime}: E_{0} \rightarrow(1 \wedge \eta)^{*} A$ in $\mathrm{EPA}_{0}$. Similarly, we can regard $(\tilde{q}, q)$ as an isomorphism

$$
\Gamma_{0}\left((\eta \wedge 1)^{*} A\right)=\left(H, T, y_{1}\right) \stackrel{\simeq}{\rightarrow}\left(G_{1}, S_{1}, x_{1}\right)=\Gamma_{0}^{*} E_{1} .
$$

As $E_{1}$ is a strong realisation of $\left(G_{1}, S_{1}, x_{1}\right)$, this comes from a map $E_{1} \rightarrow$ $(\eta \wedge 1)^{*} A$; this is easily seen to be an isomorphism, and we let $f^{\prime \prime}:(\eta \wedge 1)^{*} A \rightarrow E_{1}$ be the inverse map. It is then clear that the map

$$
f=\left(\eta^{*} f^{\prime \prime}\right) \circ\left(\eta^{*} f^{\prime}\right): \eta^{*} E_{0} \rightarrow \eta^{*} E_{1}
$$


is good and satisfies $\Gamma(f)=(\tilde{p}, p)$, so $\left(A, f^{\prime}, f^{\prime \prime}\right) \in \mathcal{B}$. Thus $\mathcal{B} \neq \emptyset$.

Now suppose we have another object $\left(B, g^{\prime}, g^{\prime \prime}\right) \in \mathcal{B}$, with $\Gamma_{1} B=\left(K, U, z_{0}, z_{1}\right)$ say. We put

$$
\begin{aligned}
& (\tilde{r}, r)=\Gamma_{1} g^{\prime \prime}:\left(G_{1}, S_{1}, x_{1}\right) \stackrel{\simeq}{\longrightarrow} \Gamma_{1}\left((\eta \wedge 1)^{*} B\right)=\left(K, U, z_{1}\right) \\
& (\tilde{s}, s)=\Gamma_{1} g^{\prime}: \Gamma_{1}\left((1 \wedge \eta)^{*} B\right)=\left(K, U, z_{0}\right) \rightarrow\left(G_{0}, S_{0}, x_{0}\right) .
\end{aligned}
$$

By hypothesis we have $(\tilde{s} \tilde{r}, s r)=(\tilde{p}, p):\left(G_{1}, s_{1}\right) \rightarrow\left(G_{0}, S_{0}\right)$. We display all these maps in the following commutative diagram:

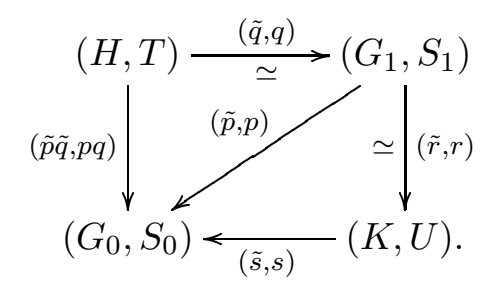

We now claim that $(\tilde{r} \tilde{q}, r q)$ can be regarded as a map

$$
\left(H, T, y_{0}, y_{1}\right) \rightarrow\left(K, U, z_{0}, z_{1}\right)
$$

Indeed, it is clear from the data recorded above that it is a map $\left(H, T, y_{1}\right) \rightarrow$ $\left(K, U, z_{1}\right)$, so it will suffice to check that $(\tilde{r} \tilde{q})^{*} z_{0}=y_{0}$. We are given that $z_{0}=\tilde{s}^{*} x_{0}$ and $\tilde{s} \tilde{r}=\tilde{p}$ and $(\tilde{p} \tilde{q})^{*} x_{0}=y_{0}$; the claim follows. As $r$ and $q$ are isomorphisms, we have an isomorphism

$$
(\tilde{r} \tilde{q}, r q)^{-1}: \Gamma_{1} B=\left(K, U, z_{0}, z_{1}\right) \rightarrow\left(H, T, y_{0}, y_{1}\right)=\Gamma_{1} A
$$

in $\mathrm{FG}_{1}$. As $A$ is a strong realization, this comes from a unique map $u: A \rightarrow B$ in $\mathrm{EPA}_{1}$, which is easily seen to be an isomorphism.

We must show that $u$ is a morphism in our category $\mathcal{B}$, or equivalently that in $\mathrm{EPA}_{0}$ we have

$$
\begin{gathered}
\left((1 \wedge \eta)^{*} u\right) f^{\prime}=g^{\prime}: E_{0} \rightarrow(1 \wedge \eta)^{*} B \\
g^{\prime \prime}\left((\eta \wedge 1)^{*} u\right)=f^{\prime \prime}:(\eta \wedge 1)^{*} B \rightarrow E_{1} .
\end{gathered}
$$

Note that $E_{0}$ and $E_{1}$ are strong, and $f^{\prime \prime}$ is an isomorphism, so $(\eta \wedge 1)^{*} B$ is strong. It is thus enough to check our two equations after applying $\pi_{0}$ (here we have used the original definition rather than the equivalent one in Defini- 
tion 3.9). By definition or construction, we have

$$
\begin{aligned}
\operatorname{spec}\left(\pi_{0} f^{\prime}\right) & =p q \\
\operatorname{spec}\left(\pi_{0} f^{\prime \prime}\right) & =q^{-1} \\
\operatorname{spec}\left(\pi_{0} g^{\prime}\right) & =s \\
\operatorname{spec}\left(\pi_{0} g^{\prime \prime}\right) & =r \\
\operatorname{spec}\left(\pi_{0} u\right) & =(r q)^{-1} \\
s r & =p .
\end{aligned}
$$

It follows easily that $\left(\pi_{0} u\right)\left(\pi_{0} f^{\prime}\right)=\pi_{0} g^{\prime}$ and $\left(\pi_{0} g^{\prime \prime}\right)\left(\pi_{0} u\right)=\pi_{0} f^{\prime \prime}$, as required. This shows that $u$ is an isomorphism in $\mathcal{B}$, and thus that $f$ is the unique good map inducing the map $(\tilde{p}, p)$.

Lemma 4.3 For any $E \in \mathcal{E}$, the identity map $1: \eta^{*} E \rightarrow \eta^{*} E$ is good.

Proof Note that the multiplication map $M P(1)=M P \wedge M P \rightarrow M P$ is a map of ring spectra (in the strict sense) and so induces a functor $\mu^{*}: \mathrm{EPA}_{0} \rightarrow \mathrm{EPA}_{1}$ with $(1 \wedge \eta)^{*} \mu^{*} E=(\eta \wedge 1)^{*} \mu^{*} E=E$ on the nose. We can thus take $A=\mu^{*} E$ and $f^{\prime}=f^{\prime \prime}=1_{E}$ to show that $1_{E}$ is good.

Proposition 4.4 Suppose we have objects $E_{0}, E_{1}, E_{2} \in \mathcal{E}$ and good morphisms $\eta^{*} E_{0} \stackrel{f}{\rightarrow} \eta^{*} E_{1} \stackrel{g}{\rightarrow} \eta^{*} E_{2}$. Then the composite $g f$ is also good.

Proof Write $\left(G_{i}, S_{i}, x_{i}\right)=\Gamma_{0} E_{i}$ and $(\tilde{p}, p)=\Gamma(f):\left(G_{1}, S_{1}\right) \rightarrow\left(G_{0}, S_{0}\right)$ and $(\tilde{q}, q)=\Gamma(g):\left(G_{2}, S_{2}\right) \rightarrow\left(G_{1}, S_{1}\right)$.

Choose objects $A, B \in \mathrm{EPA}_{1}$ and maps

$$
\begin{gathered}
f^{\prime}: E_{0} \rightarrow(1 \wedge \eta)^{*} A \\
f^{\prime \prime}:(\eta \wedge 1)^{*} A \stackrel{\simeq}{\longrightarrow} E_{1} \\
g^{\prime}: E_{1} \rightarrow(1 \wedge \eta)^{*} B \\
g^{\prime \prime}:(\eta \wedge 1)^{*} B \stackrel{\simeq}{\longrightarrow} E_{2}
\end{gathered}
$$

exhibiting the goodness of $f$ and $g$. This gives rise to isomorphisms

$$
\begin{aligned}
& \Gamma_{1} A=\left(G_{1}, S_{1}, \tilde{p}^{*} x_{0}, x_{1}\right) \\
& \Gamma_{1} B=\left(G_{2}, S_{2}, \tilde{q}^{*} x_{1}, x_{2}\right) .
\end{aligned}
$$

Next, observe that we have an object $\left(G_{2}, S_{2},(\tilde{p} \tilde{q})^{*} x_{0}, \tilde{q}^{*} x_{1}, x_{2}\right) \in \mathrm{FG}_{2}$, which is strongly realisable because $x_{2}$ is a multirealisable coordinate. We can thus choose an object $P \in \mathrm{EPA}_{2}$ and an isomorphism

$$
(\tilde{r}, r): \Gamma_{2} P \rightarrow\left(G_{2}, S_{2},(\tilde{p} \tilde{q})^{*} x_{0}, \tilde{q}^{*} x_{1}, x_{2}\right)
$$


making $P$ a strong realisation. We can also regard $(\tilde{r}, r)$ as an isomorphism

$$
\Gamma_{1}\left((\eta \wedge 1 \wedge 1)^{*} P\right) \rightarrow\left(G_{2}, S_{2}, \tilde{q}^{*} x_{1}, x_{2}\right)=\Gamma_{1} B
$$

As $B$ is strong, this comes from a unique isomorphism $v:(\eta \wedge 1 \wedge 1)^{*} P \rightarrow B$ in $\mathrm{EPA}_{1}$.

Similarly, we can regard $(\tilde{r}, r)$ as an isomorphism

$$
\Gamma_{1}\left((1 \wedge 1 \wedge \eta)^{*} P\right) \rightarrow\left(G_{2}, S_{2}, \tilde{q}^{*} \tilde{p}^{*} x_{0}, \tilde{q}^{*} x_{1}\right),
$$

and we can regard $(\tilde{q}, q)$ as a morphism

$$
\left(G_{2}, S_{2}, \tilde{q}^{*} \tilde{p}^{*} x_{0}, \tilde{q}^{*} x_{1}\right) \rightarrow\left(G_{1}, S_{1}, \tilde{p}^{*} x_{0}, x_{1}\right) \simeq \Gamma_{1} A .
$$

As $A$ is strong, the composite $(\tilde{q} \tilde{r}, q r)$ must come from a unique map $u: A \rightarrow$ $(1 \wedge 1 \wedge \eta)^{*} P$ in $\mathrm{EPA}_{1}$.

We now put

$$
\begin{aligned}
C & =(1 \wedge \eta \wedge 1)^{*} P \in \mathrm{EPA}_{1} \\
h^{\prime} & =\left(E_{0} \stackrel{f^{\prime}}{\longrightarrow}(1 \wedge \eta)^{*} A \stackrel{(1 \wedge \eta)^{*} u}{\longrightarrow}(1 \wedge \eta \wedge \eta)^{*} P=(1 \wedge \eta)^{*} C\right) \\
h^{\prime \prime} & =\left((\eta \wedge 1)^{*} C=(\eta \wedge \eta \wedge 1)^{*} P \stackrel{(\eta \wedge 1)^{*} v}{\longrightarrow}(\eta \wedge 1)^{*} B \stackrel{g^{\prime \prime}}{\longrightarrow} E_{2}\right) .
\end{aligned}
$$

As $v$ and $g^{\prime \prime}$ are isomorphisms, the same is true of $h^{\prime \prime}$. We claim that after forgetting down to EPR, we have $h^{\prime \prime} h^{\prime}=g f$; this will prove that $g f$ is good as claimed. We certainly have $h^{\prime \prime} h^{\prime}=g^{\prime \prime} v u f^{\prime}$ and $g f=g^{\prime \prime} g^{\prime} f^{\prime \prime} f^{\prime}$ so it will suffice to show that $v u=g^{\prime} f^{\prime \prime}: A \rightarrow B$ in EPR. For this, it will be enough to prove that the following diagram in $\mathrm{EPA}_{0}$ commutes.

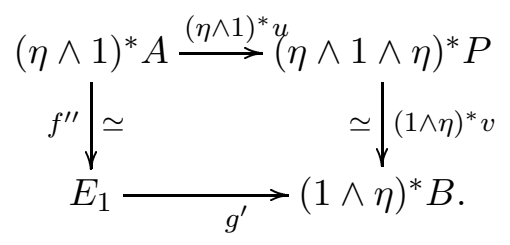

As this is a diagram in $\mathrm{EPA}_{0}$ and $(\eta \wedge 1)^{*} A \simeq E_{1}$ is strong, it will be enough to check that the diagram commutes after applying $\pi_{0}$. By construction we have $\pi_{0}(u)=w^{-1} \circ \psi \circ \pi_{0}\left(f^{\prime \prime}\right)$ and $\psi=\pi_{0}(g)=\pi_{0}\left(g^{\prime \prime}\right) \circ \pi_{0}\left(g^{\prime}\right)$ and $\pi_{0}(v)=$ $\pi_{0}\left(g^{\prime \prime}\right)^{-1} \circ w$. It follows directly that the above diagram commutes on homotopy, groups, so it commutes in $\mathrm{EPA}_{0}$, so it commutes in EPR, so $g f=h^{\prime \prime} h^{\prime}$ in EPR as explained previously. Thus, the map $g f$ is good, as claimed.

Proof of Theorem 1.6 We merely need to collect results together and explain the argument in the introduction in more detail. Lemma 4.3 and Proposition 4.4 show that we can make $\mathcal{E}$ into a category by taking the good maps 
from $\eta^{*} E_{0}$ to $\eta^{*} E_{1}$ as the morphisms from $E_{0}$ to $E_{1}$. Tautologically, we can define a faithful functor $U: \mathcal{E} \rightarrow$ EPR by $U(E)=\eta^{*} E$ and $U(f)=f$. We then define $V=\Gamma U: \mathcal{E} \rightarrow \mathrm{FG}$; by the definition of $\mathcal{E}$, this actually lands in GFG. Proposition 4.1 says that $V$ is essentially surjective, and Proposition 4.2 says that $V$ is full and faithful. This means that $V$ is an equivalence, so we can invert it and define $R=U V^{-1}:$ GFG $\rightarrow \mathrm{EPR}$. As $V=\Gamma U$ we have $\Gamma R=1$, so $R$ is the required realisation functor.

\section{A Appendix : The product on $M P$}

In this appendix we verify that $M P$ can be constructed as an $E_{\infty}$ ring spectrum.

Let $\mathcal{U}$ be a complex universe. For any finite-dimensional subspace $U$ of $\mathcal{U}$, we write $U_{L}=U \oplus 0<\mathcal{U} \oplus \mathcal{U}$ and $U_{R}=0 \oplus U<\mathcal{U} \oplus \mathcal{U}$. We let $\operatorname{Grass}(U \oplus U)$ denote the Grassmannian of all subspaces of $U \oplus U$ (of all possible dimensions), and we write $\gamma_{U}$ for the tautological bundle over this space, and $\operatorname{Thom}(U \oplus U)$ for the associated Thom space. If $U \leq U^{\prime}<\mathcal{U}$ then we define $i$ : $\operatorname{Grass}\left(U^{2}\right) \rightarrow$ $\operatorname{Grass}\left(\left(U^{\prime}\right)^{2}\right)$ by $i(A)=A \oplus\left(U^{\prime} \ominus U\right)_{R}$. On passing to Thom spaces we get a map $\sigma: \Sigma^{U^{\prime} \ominus U} \operatorname{Thom}\left(U^{2}\right) \rightarrow \operatorname{Thom}\left(\left(U^{\prime}\right)^{2}\right)$. These maps can be used to assemble the spaces Thom $\left(U^{2}\right)$ into a $\Sigma$-inclusion prespectrum indexed by the complex subspaces of $\mathcal{U}$. We write $T_{\mathcal{U}}$ for this prespectrum, and $M P_{\mathcal{U}}$ for its spectrification.

Now let $\mathcal{V}$ be another complex universe, so we have a prespectrum $T_{\mathcal{V}}$ over $\mathcal{V}$, and thus an external smash product $T_{\mathcal{U}} \wedge_{\text {ext }} T_{\mathcal{V}}$ indexed on the complex subspaces of $\mathcal{U} \oplus \mathcal{V}$ of the form $U \oplus V$. The direct sum gives a map $\operatorname{Grass}\left(U^{2}\right) \times$ $\operatorname{Grass}\left(V^{2}\right) \rightarrow \operatorname{Grass}\left((U \oplus V)^{2}\right)$ which induces a map $\operatorname{Thom}\left(U^{2}\right) \wedge \operatorname{Thom}\left(V^{2}\right) \rightarrow$ $\operatorname{Thom}\left((U \oplus V)^{2}\right)$. These maps fit together to give a map $T_{\mathcal{U}} \wedge_{\text {ext }} T_{\mathcal{V}} \rightarrow T_{\mathcal{U} \oplus \mathcal{V}}$ and thus a map $M P_{\mathcal{U}} \wedge_{\text {ext }} M P_{\mathcal{V}} \rightarrow M P_{\mathcal{U} \oplus \mathcal{V}}$ of spectra over $\mathcal{U} \oplus \mathcal{V}$. Essentially the same construction gives maps

$$
M P_{\mathcal{U}_{1}} \wedge_{\text {ext }} \cdots \wedge_{\text {ext }} M P_{\mathcal{U}_{r}} \rightarrow M P_{\mathcal{U}_{1} \oplus \ldots \oplus \mathcal{U}_{r}} .
$$

If $\mathcal{U}_{1}=\ldots \mathcal{U}_{r}=\mathcal{U}$, then this map is $\Sigma_{r}$-equivariant.

Now suppose instead that we have a complex linear isometry $f: \mathcal{U} \rightarrow \mathcal{V}$. This gives evident homeomorphisms $\operatorname{Thom}\left(U^{2}\right) \rightarrow \operatorname{Thom}\left((f U)^{2}\right)$, which fit together to induce a map $M P_{\mathcal{U}} \rightarrow f^{*} M P_{\mathcal{V}}$, which is adjoint to a map $f_{*} M P_{\mathcal{U}} \rightarrow M P_{\mathcal{V}}$. We next observe that this construction is continuous in all possible variables, including $f$. (This statement requires some interpretation, but there are no new 
issues beyond those that are well-understood for $M U$; the cleanest technical framework is provided by [1].) It follows that they fit together to give a map $\mathcal{L}_{\mathbb{C}}(\mathcal{U}, \mathcal{V}) \ltimes M P_{\mathcal{U}} \rightarrow M P_{\mathcal{V}}$ of spectra over $\mathcal{V}$.

We now combine this with the product structure mentioned earlier to get a map

$$
\mathcal{L}_{\mathbb{C}}\left(\mathcal{U}^{r}, \mathcal{U}\right) \ltimes_{\Sigma_{r}}\left(M P_{\mathcal{U}} \wedge_{\text {ext }} \ldots \wedge_{\text {ext }} M P_{\mathcal{U}}\right) \rightarrow M P_{\mathcal{U}}
$$

This means that $M P_{\mathcal{U}}$ has an action of the $E_{\infty}$ operad of complex linear isometries, as required.

All that is left is to check that the spectrum $M P=M P_{\mathbb{C}^{\infty}}$ constructed above has the right homotopy type. As $T$ is a $\Sigma$-inclusion prespectrum, we know that spectrification works in the simplest possible way and that $M P$ is the homotopy colimit of the spectra

$$
\Sigma^{-2 n} \operatorname{Thom}\left(\mathbb{C}^{n} \oplus \mathbb{C}^{n}\right)=\bigvee_{k=-n}^{n} \Sigma^{-2 n} \operatorname{Grass}_{k+n}\left(\mathbb{C}^{n} \oplus \mathbb{C}^{n}\right)^{\gamma},
$$

where $\operatorname{Grass}_{d}(V)$ is the space of $d$-dimensional subspaces of $V$. It is not hard to see that the maps of the colimit system preserve this splitting, so that $M P$ is the wedge over all $k \in \mathbb{Z}$ of the spectra

$$
X_{k}:=\underset{n}{\operatorname{holim}} \Sigma^{-2 n} \operatorname{Grass}_{k+n}\left(\mathbb{C}^{n} \oplus \mathbb{C}^{n}\right)^{\gamma}
$$

This can be rewritten as

$$
X_{k}=\Sigma^{2 k} \underset{n, m}{\operatorname{holim}} \Sigma^{-2(k+n)} \operatorname{Grass}_{k+n}\left(\mathbb{C}^{m} \oplus \mathbb{C}^{n}\right)^{\gamma} .
$$

We can reindex by putting $n=i-k$ and $m=j+k$, and then pass to the limit in $j$. We find that

$$
X_{k}=\Sigma^{2 k} \underset{i}{\operatorname{holim}} \Sigma^{-2 i} \operatorname{Grass}_{i}\left(\mathbb{C}^{\infty} \oplus \mathbb{C}^{i}\right)^{\gamma} .
$$

It is well-known that $\operatorname{Grass}_{i}\left(\mathbb{C}^{\infty} \oplus \mathbb{C}^{i}\right)$ is a model for $B U(i)$, and it follows that $X_{k}=\Sigma^{2 k} M U$, so $M P=\bigvee_{k} \Sigma^{2 k} M U$ as claimed. We leave it to the reader to check that the product structure is the obvious one.

All the above was done without inverting 2. Inverting 2 is an example of Bousfield localisation, and this can always be performed in the category of strictly commutative ring spectra. 


\section{References}

[1] A D Elmendorf, The Grassmannian Geometry of Spectra, Journal of Pure and Applied Algebra 54 (1988) 37-94

[2] A D Elmendorf, I Kriz, M A Mandell, J P May, Rings, Modules and Algebras in Stable Homotopy Theory, volume 47 of Amer. Math. Soc. Surveys and Monographs, American Mathematical Society (1996)

[3] L Gaunce Lewis, J Peter May, M Steinberger (with contributions by Jim E McClure), Equivariant Stable Homotopy Theory, volume 1213 of Lecture Notes in Mathematics, Springer-Verlag, New York (1986)

[4] N P Strickland, Products on MU-modules, Trans. Amer. Math. Soc. 351 (1999) 2569-2606

[5] Neil P Strickland, Formal schemes and formal groups, from: "Homotopy invariant algebraic structures (Baltimore, MD, 1998)", Amer. Math. Soc., Providence, RI (1999) 263-352

Department of Pure Mathematics, University of Sheffield

Sheffield S3 7RH, UK

Email: N.P.Strickland@sheffield.ac.uk

Received: 9 January 2003 